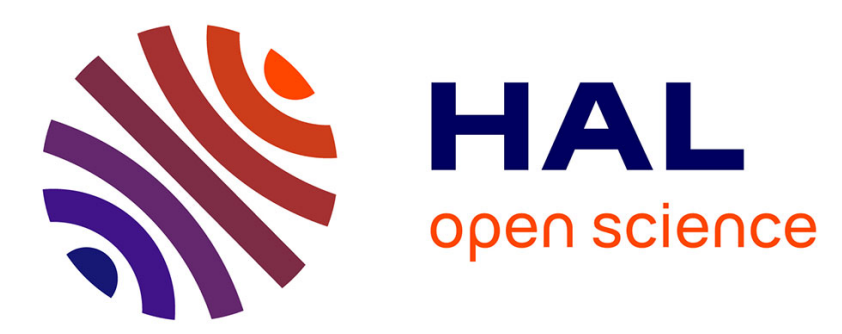

\title{
Influence of the microstructure texture of nickel base and cobalt base cast superalloys on their oxdation behaviour at high temperature
}

Patrice Berthod, Steven Raude, Aurélie Chiaravalle, Anne-Sophie Renck, Christophe Rapin, Renaud Podor

\section{To cite this version:}

Patrice Berthod, Steven Raude, Aurélie Chiaravalle, Anne-Sophie Renck, Christophe Rapin, et al.. Influence of the microstructure texture of nickel base and cobalt base cast superalloys on their oxdation behaviour at high temperature. Revue de Métallurgie, 2004, 101 (12), pp.1031-1042. 10.1051/metal:2004109 . hal-02108983

\section{HAL Id: hal-02108983 https://hal.science/hal-02108983}

Submitted on 30 Apr 2019

HAL is a multi-disciplinary open access archive for the deposit and dissemination of scientific research documents, whether they are published or not. The documents may come from teaching and research institutions in France or abroad, or from public or private research centers.
L'archive ouverte pluridisciplinaire HAL, est destinée au dépôt et à la diffusion de documents scientifiques de niveau recherche, publiés ou non, émanant des établissements d'enseignement et de recherche français ou étrangers, des laboratoires publics ou privés. 


\title{
Influence de la texture microstructurale de superalliages de fonderie à base de nickel et à base de cobalt sur leur comportement en oxydation à haute température
}

\author{
P. Berthod, S. Raude, A. Chiaravalle, A.-S. Renck, \\ C. Rapin, R. Podor \\ Laboratoire de Chimie du Solide Minéral (U.M.R. 7555), \\ Faculté des Sciences et Techniques, Université Henri Poincaré Nancy 1, \\ Vandœuvre-les-Nancy
}

\begin{abstract}
Le comportement en oxydation à haute température de superalliages de fonderie renforcés par carbures a été étudiée en fonction de leur finesse de grains et de leur orientation dendritique en surface. Ces deux caractéristiques microstructurales ont montré chacune une influence sensible sur la cinétique d'oxydation, dans un sens qui dépend de l'élément de base du superalliage, de sa composition ou de la nature de ses carbures.
\end{abstract}

\section{INTRODUCTION}

Les turbines aéronautiques ou terrestres (aubes), comme certains procédés industriels travaillant à chaud (travail du verre fondu), font appel à des superalliages de fonderie comme matériaux constitutifs de leurs pièces les plus exposées à de hautes températures. En plus de leur composition chimique, certaines caractéristiques microstructurales des alliages de fonderie sont susceptibles d'avoir une influence sur leur résistance à l'oxydation à haute température : ce sont la finesse microstructurale et l'orientation granulaire. En effet, les phénomènes d'oxydation dépendent de processus diffusionnels au travers de l'oxyde déjà formé mais aussi au travers de la zone la plus extérieure de l'alliage lui-même.

Manuscrit reçu le 3 novembre 2003, bon à publier le 22 novembre 2004.

(C) La Revue de Métallurgie 2004.
Dans l'alliage, la diffusion des espèces atomiques ou ioniques impliquées dans le processus d'oxydation peut se faire au travers de la matrice, des joints de grains ou des oxydes internes déjà formés, avec un rôle souvent important des joints de grains (1). Ces derniers représentent des chemins de diffusion privilégiés. De plus, ils contiennent souvent des carbures primaires apparus en fin de solidification formant un eutectique avec la matrice et contenant des éléments particulièrement oxydables tels que chrome, tungstène et tantale. Morphologie et orientation des joints de grains apparaissent alors susceptibles d'avoir une influence sur la cinétique d'oxydation globale de l'alliage.

Cette étude tente de préciser cette influence possible de la texture microstructurale sur la cinétique d'oxydation à haute température, pour plusieurs superalliages de fonderie de natures diverses et de caractéristiques microstructurales bien choisies en termes de finesse et d'orientation.

\section{- RAPPELS SUR LES RELATIONS SOLIDIFICATION - MICROSTRUCTURE}

La solidification d'alliages de fonderie classique, métalliques et polycristallins, est caractérisée par une forte dépendance de la taille de grain et de l'orientation granulaire (dendritique dans le cas des alliages à base de nickel et à base de cobalt) vis-à-vis respectivement de la vitesse de solidification et du gradient thermique qui existent localement pendant la solidification $(2,3,4)$. Ceci est illustré par la figure 1 schématisant la microstructure d'une pièce polycristalline moulée classique (avec les trois zones que l'on observe habituellement dans ce cas).

Lorsque la vitesse de solidification est lente, comme dans le cas de pièces massives (taille décimétrique et plus) coulées en moule de sable lié, la microstructure obtenue est caractérisée par de gros grains. Quand, au contraire, la solidification est plus rapide (pièces d'épaisseur centimétrique et moins, utilisation de moules métalliques,...), les grains obtenus sont plus nombreux et de taille plus petite, d'où une microstructure fine. 


\title{
Influence of the microstructure texture of nickel base and cobalt base cast superalloys on their oxidation behaviour at high temperature
}

\author{
P. Berthod, S. Raude, A. Chiaravalle, A.-S. Renck, \\ C. Rapin, R. Podor \\ Laboratoire de Chimie du Solide Minéral (U.M.R. 7555), \\ Faculté des Sciences et Techniques, Université Henri Poincaré Nancy 1, \\ Vandœuvre-les-Nancy
}

Solidification conditions induce microstructural characteristics of cast alloys. Grains can be coarse or fine if the cooling rate was slow or fast. Grains can be also oriented or equiaxed, depending if there was a marked thermal gradient or not. In the first case, growth direction, then dendrite orientation, is mainly parallel to this thermal gradient. Grain boundaries are of a great importance on the high temperature oxidation behaviour of cast superalloys since they act as short diffusion paths for the elements involved in the oxidation phenomena (Cr for example). Therefore, morphology (density, thickness) and mean orientation of grain boundaries may notably influence the oxidation rate of these alloys.

To better analyze the influence of these microstructural features on oxidation behaviour, selected superalloys were synthesized and tested in oxidation at $1000^{\circ} \mathrm{C}$ and higher temperatures using a thermogravimetry technique. Parabolic rate constants were determined and surface microstructures were examined. To study the influence of the grain fineness, four carbide strengthened superalloys, one nickel base and three cobalt base, were synthesized by sand mould castings and by cold crucible castings. Then they get respectively coarse and fine microstructures (about 60 and $20 \mu \mathrm{m}$ for the apparent grain sizes). The oxidation rate effectively depend of the grain fineness. But, for two of these alloys, a fine microstructure led to a faster oxidation than for a coarser microstructure. The opposite effect is observed for the two other alloys. The main difference between these two couples of superalloys is that the first alloys contain tungsten in their chemical composition while this element is not present in the other alloys. One other interesting observation is that sensible differences exist between alloys of same coarse microstructure (oxidation rate raises with carbon content for example), but all oxidation rates are nearly equals when alloys have a finer microstructure.

For the dendritic orientation part of this study, only two superalloys were considered, the nickel base one and one of the cobalt base alloys. Samples for thermogravimetry trials were especially prepared, in order to maximize either the surface which is mainly parallel to dendrites (more precisely to their primary arms) or the one which is mainly perpendicular to dendrites. Oxidation trials first indicate that, for a same alloy, there is a sensible difference of oxidation rate between the two types of sample. Moreover, after a mathematical uncoupling of the contributions of the faces which are parallel to dendrites and the ones which are perpendicular, differences between the two orientations became more marked. The nickel base alloy oxidizes faster when its surface is perpendicular to dendrites than it is parallel. It is the opposite phenomena for the cobalt base alloy, but difference is smaller for that one.

To conclude, grain size and dendritic orientation seem to have a real effect on the oxidation rate. It must be certainly related to the specific role of grain boundaries on the diffusion of chromium and of other elements involved in the oxidation phenomena. It could also be thought that the external chromia scale presents different structures or amounts of defects, depending of alloys grain size and grain boundaries orientation. Deeper investigations have to be performed to determine how these texture parameters exactly act. The dependence of the oxidation rates on chemical composition and the presumed role of elements like tungsten also remain to be explained. For an industrial use of these results, the role of the grain size is the most interesting, since controlling the dendritic orientation of a cast piece along its external surface is not easy. It is especially true if it is the parallel orientation which displays the best oxidation behaviour. On the other hand, knowing this influence can induce better method for characterizing oxidation rate of alloys, by specific cutting of samples, to take into account a possible effect of the microstructural orientation, as already made for mechanical tests for example. 


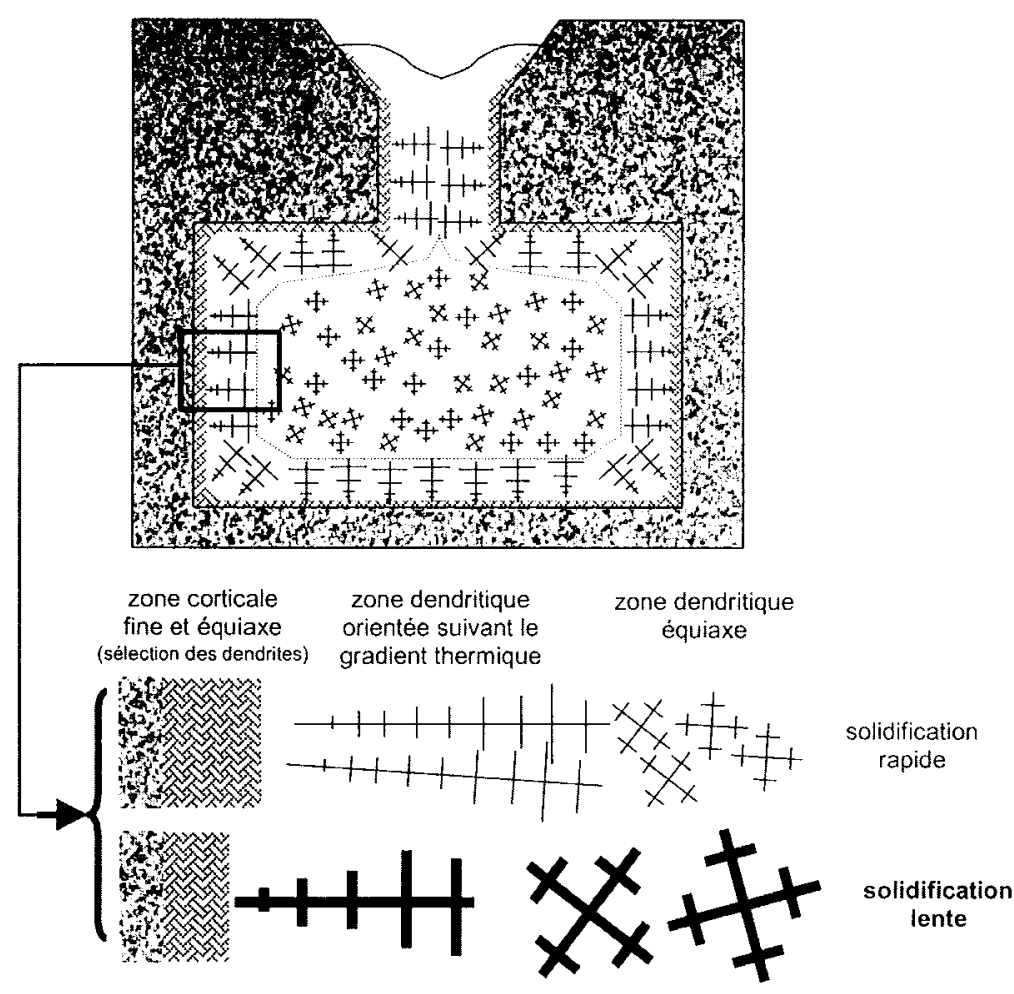

Figure 1 - Schéma de la microstructure d'une pièce moulée: les trois zones successives, finesse microstructurale fonction de la vitesse de solidification et orientation dendritique suivant le gradient thermique local.

Figure 1 -Scheme of the microstructure of a cast piece : the three successive zones, microstructure fineness versus solidification rate and dendritic orientation depending of the local thermal gradient.

La croissance des dendrites est d'autant plus rapide qu'elles sont mieux orientées par rapport au gradient thermique local. Les dendrites les mieux orientées croissent rapidement et bloquent la croissance des autres dendrites. Depuis la paroi du moule et en direction du centre de la pièce, on rencontre ainsi généralement une fine couche équiaxe corticale, puis une zone où les dendrites sont toutes orientées vers le centre thermique de la pièce. Si la pièce est massive, la solidification de la dernière zone se fait avec croissance équiaxe des dendrites, du fait de l'absence de gradient thermique vraiment marqué. Suivant sa taille, la technique de moulage utilisée et l'usinage réalisé sur le brut, la pièce finale présente localement des surfaces où la microstructure est fine ou grossière, parallèle ou perpendiculaire à cette surface.

\section{ÉTUDE DE L'INFLUENCE DE LA FINESSE MICROSTRUCTURALE}

\section{Détails expérimentaux}

Les alliages de cette étude sont quatre superalliages de fonderie renforcés par carbures, dont le tableau / donne les compositions chimiques. Du fait de leur réfractairité $\left(T_{\text {solidus }}>1200^{\circ} \mathrm{C}\right)$, de leur haute résistance mécanique (présence de carbures) et de leur résistance à l'oxydation et à la corrosion à chaud $(30 \%$ massiques de chrome), ce sont des alliages pouvant être utilisés comme matériaux constitutifs de pièces industrielles fonctionnant en atmosphère oxydante à haute température. L'alliage nommé "BN1 " est un superalliage à base de nickel présentant des carbures de type $(\mathrm{Cr}, \mathrm{W})_{23} \mathrm{C}_{6}$ eutectiques dans les joints interdendritiques. Les alliages « $\mathrm{BC} 1$ », « $\mathrm{BC} 2$ » et « $\mathrm{BC} 3$ » sont des superalliages à base de cobalt contenant des carbures eutectiques de diverses natures: carbures de chrome ou chrome/tungstène $(\mathrm{Cr}, \mathrm{W})_{23} \mathrm{C}_{6}$ et carbures de tantale TaC.

Ces alliages ont été élaborés initialement à partir d'éléments de pureté minimale $99 \%$ en masse pour Co (fournisseur : Ufaleynickel), $\mathrm{Cr}$ (Delachaux) et $\mathrm{Fe}$ (Armco) et $99,9 \%$ pour $\mathrm{Ni}$ (Eramet), Ta (Aerometal) et $\mathrm{C}$ (graphite). Dans un premier temps, les fusions ont été opérées dans un four à induction de plusieurs kilogrammes de capacité sous atmosphère d'argon. Les alliages sont ensuite solidifiés en moule de sable lié, sous forme de pièces moulées de taille caractéristique d'environ $3 \mathrm{~cm}$ (épaisseur moyenne), d'où une solidification assez lente. Des plaquettes de thermogravimétrie, dites de microstructure grossière, ont d'abord été prélevées dans ces premières pièces. Dans un second temps, des morceaux d'environ $30 \mathrm{~g}$ ont été prélevés au centre des pièces précédentes, et refondus au moyen d'un four de fusion HF sur sole froide (en cuivre, refroidie à l'eau), toujours sous atmosphère inerte d'argon. Les alliages sont solidifiés dans ce même creuset refroidi, après coupure de l'alimentation. C'est ainsi qu'ont été obtenues les microstructures fines, avec les mêmes compositions chimiques que les microstructures grossières.

TABLEAU I - Composition chimique des alliages étudiés (partie finesse microstructurale).

TABLE I - Chemical composition of the studied alloys (fineness part).

\begin{tabular}{|c|c|c|c|c|c|c|c|}
\hline $\begin{array}{r}\% \text { mass. } \\
\% \text { atom. }\end{array}$ & $\mathrm{Co}$ & $\mathrm{Ni}$ & $\mathrm{Cr}$ & $\mathrm{C}$ & $\mathrm{W}$ & $\mathrm{Ta}$ & $\mathrm{Fe}$ \\
\hline \multirow{2}{*}{$\mathrm{BC} 1$} & 53,3 & 8,5 & 29,0 & 0,38 & 5,8 & 3,0 & $/$ \\
& 53,63 & 8,58 & 33,06 & 1,88 & 1,87 & 0,98 & $/$ \\
\hline \multirow{2}{*}{$\mathrm{BC} 2$} & 57,0 & 8,5 & 28,3 & 0,37 & $/$ & 5,8 & $/$ \\
& 56,27 & 8,42 & 31,65 & 1,79 & $/$ & 1,86 & $/$ \\
\hline \multirow{2}{*}{$\mathrm{BC} 3$} & 55,7 & 8,5 & 29,0 & 0,98 & $/$ & 5,8 & $/$ \\
& 53,67 & 8,22 & 31,66 & 4,63 & $/$ & 1,82 & $/$ \\
\hline \multirow{2}{*}{$\mathrm{BN} 1$} & $/$ & 55,8 & 28,5 & 0,70 & 7,5 & $/$ & 7,5 \\
& $/$ & 54,89 & 31,64 & 3,36 & 2,35 & $/$ & 7,75 \\
\hline
\end{tabular}




\section{oxydation}

Les plaquettes pour essai thermogravimétrique sont d'une masse d'environ $1,5 \mathrm{~g}$ et de forme parallélépipédique, avec une surface extérieure d'environ $2 \mathrm{~cm}^{2}$. Elles ont été prélevées au cœur des lingots, dans la zone de solidification équiaxe. Les plaquettes ont été polies sur leurs six faces jusqu'au papier 1200 , avec arrondissement des arêtes et sommets. Les tests thermogravimétriques d'oxydation à haute température ont été réalisés dans une thermobalance Setaram TG92, sous circulation d'air synthétique sec $(80 \%$ $\mathrm{N}_{2}-20 \% \mathrm{O}_{2}$ d'Air Liquide). Le cycle est composé d'un chauffage à la vitesse de $20^{\circ} \mathrm{C} / \mathrm{min}$ jusqu'à la température d'essai $\left(1000\right.$ ou $1100^{\circ} \mathrm{C}$ ), d'un palier isotherme de $100 \mathrm{~h}$, puis d'un refroidissement à la vitesse de $10^{\circ} \mathrm{C} / \mathrm{min}$. Les plaquettes ont ensuite été revêtues d'un dépôt électrolytique de nickel destiné à protéger la couche d'oxydes externe. Elles ont ensuite été découpées, enrobées et polies jusqu'au feutre $3 \mu \mathrm{m}$. Les observations métallographiques ont été faites essentiellement avec un Microscope Électronique à Balayage (MEB) de type Philips XL30, en mode Électrons Rétrodiffusés ("Back Scattered Electrons », BSE) sous une tension d'accélération de $20 \mathrm{kV}$. Le contrôle des compositions d'alliage et de leur homogénéité chimique, tous éléments carbone excepté, ont été réalisés en Spectrométrie de Dispersion d'Énergie (EDS) sur des plages entières à $\times 125$ et $\times 250$. Les compositions des oxydes formés, externes ou internes, ont été déterminées au MEB en mode EDS ponctuel.

Les fichiers numériques des valeurs de prise de masse en fonction du temps sont traités à l'aide d'un tableur afin de calculer la valeur de la constante parabolique d'oxydation $\mathrm{Kp}$ à partir de l'expression de la vitesse d'oxydation :

$$
\frac{\mathrm{d}\left(\frac{\Delta \mathrm{m}}{\mathrm{S}}\right)}{\mathrm{dt}}=\frac{\mathrm{K}_{\mathrm{p}}}{\left(\frac{\Delta \mathrm{m}}{\mathrm{S}}\right)}
$$

ou de la cinétique d'oxydation qui en est déduite :

$$
\frac{\Delta \mathrm{m}}{\mathrm{S}}(\mathrm{t})=\sqrt{2 \cdot \mathrm{K}_{\mathrm{p}}} \cdot \mathrm{t}^{\frac{1}{2}}
$$

$\Delta m / S$ est la prise de masse par unité d'aire de surface résultant de la formation puis de la croissance de la couche continue d'oxyde de chrome $\mathrm{Cr}_{2} \mathrm{O}_{3}$ (chromine) en surface. Quand elle a lieu, l'oxydation interne d'éléments d'alliage est également comptabilisée dans cette prise de masse. Cette loi cinétique en racine carrée du temps résulte du rôle prépondérant des phénomènes de diffusion au travers de la couche d'oxyde externe elle-même. Le ralentissement de la vitesse d'oxydation avec le temps traduit l'effet protecteur croissant de la couche d'oxyde devenant plus épaisse.

L'oxyde de chrome, $\mathrm{Cr}_{2} \mathrm{O}_{3}$ en dessous de $1000^{\circ} \mathrm{C}$, devient volatil sous la forme $\mathrm{CrO}_{3}$ à plus haute température et quitte la surface de l'échantillon. Afin de mieux représenter la progression de l'oxydation par le suivi de gain de masse à $\mathrm{T}>1000^{\circ} \mathrm{C}$, il a été additionné, aux valeurs $[\Delta \mathrm{m} / \mathrm{S}] \cdot(\mathrm{t})$ mesurées, un terme en Kv.t où $\mathrm{Kv}$, constante de volatilisa- tion, a été prise égale à $0,45 \cdot 10^{-10} \mathrm{~g} \cdot \mathrm{cm}^{-2} \cdot \mathrm{s}^{-1}$ pour $1050^{\circ} \mathrm{C}$ et $2,35.10^{-10} \mathrm{~g} \cdot \mathrm{cm}^{-2} \cdot \mathrm{s}^{-1}$ pour $1100^{\circ} \mathrm{C}$, avant traitement des fichiers résultats des essais en vue de la détermination des constantes $\mathrm{Kp}$. Ces valeurs proviennent de constantes de volatilisation antérieurement déterminées sur des alliages très proches de ceux étudiés ici (7).

D'une manière générale, l'oxydation ayant eu lieu au cours de la montée en température, sur une durée de 5 à $10 \mathrm{~min}$ de séjour entre $900^{\circ} \mathrm{C}$ et la température de palier (températures où la vitesse d'oxydation est significative pour ce type d'alliages), peut être considérée comme très limitée. De même le gain de masse pendant le régime transitoire de l'oxydation en début de palier (dans la grande majorité des cas sur un maximum de $5 \mathrm{~h}$ ), a peu contribué à la prise de masse sur les courbes obtenues. Ces dernières sont toutes d'allure parabolique, au moins sur la première moitié de l'essai pour celles qui sont affectées par un décollement de la couche d'oxyde. De ce fait, le tracé $\Delta \mathrm{m} / \mathrm{S}$ en fonction de $\mathrm{t}^{1 / 2}$, origine du temps et origine de la masse étant prises au début du palier, conduit à une courbe généralement rectiligne et passant quasiment par l'origine du repère. La valeur de $\mathrm{Kp}$ est donc déterminée à partie de la pente de cette courbe.

\section{Microstructures des alliages}

La figure 2 présente une sélection de micrographies illustrant la diversité des microstructures et donnant un exemple de l'affinement de microstructure obtenu par une solidification rapide.

L'alliage à base de cobalt $\mathrm{BC} 1$ possède une matrice dendritique contenant d'autres éléments en solution solide (essentiellement $\mathrm{Ni}, \mathrm{Cr}$ et $\mathrm{W}$ ) et des carbures interdendritiques de deux types ( $\mathrm{TaC}$ et $(\mathrm{Cr}, \mathrm{W})_{23} \mathrm{C}_{6}$ ) formant chacun un eutectique avec la matrice. L'alliage $\mathrm{BC} 2$ présente également une matrice dendritique et un réseau assez dense de carbures $\mathrm{TaC}$ eutectiques dans les joints interdendritiques. L'alliage BC3 se caractérise par un réseau très dense de carbures interdendritiques, constitué pour moitié de $\mathrm{Cr}_{7} \mathrm{C}_{3}$ eutectiques et pour moitié de $\mathrm{TaC}$ eutectiques. Enfin, I'alliage à base de nickel $\mathrm{BN} 1$ possède une matrice dendritique (incluant les éléments $\mathrm{Cr}, \mathrm{W}, \mathrm{C}, \mathrm{Fe}$ en solution solide) et un réseau dense de carbures $(\mathrm{Cr}, \mathrm{W})_{23} \mathrm{C}_{6}$. Ces carbures confèrent aux différents alliages des propriétés de résistance mécanique à haute température remarquables, notamment à la déformation par fluage $(5,6)$.

Solidifiés en moules de sable, les alliages ont une taille de grain d'une cinquantaine de micromètres, tandis qu'elle n'est que d'une vingtaine de micromètres lorsqu'ils sont solidifiés dans le creuset refroidi.

Compte tenu de la rapidité de la refusion en four HF (moins de $3 \mathrm{~min}$ pour fusion et solidification) et de l'atmosphère d'argon, les compositions chimiques des alliages n'ont pas sensiblement évolué durant cette opération et sont quasiment identiques entre un alliage à microstructure grossière et le même alliage avec microstructure fine (à la précision 

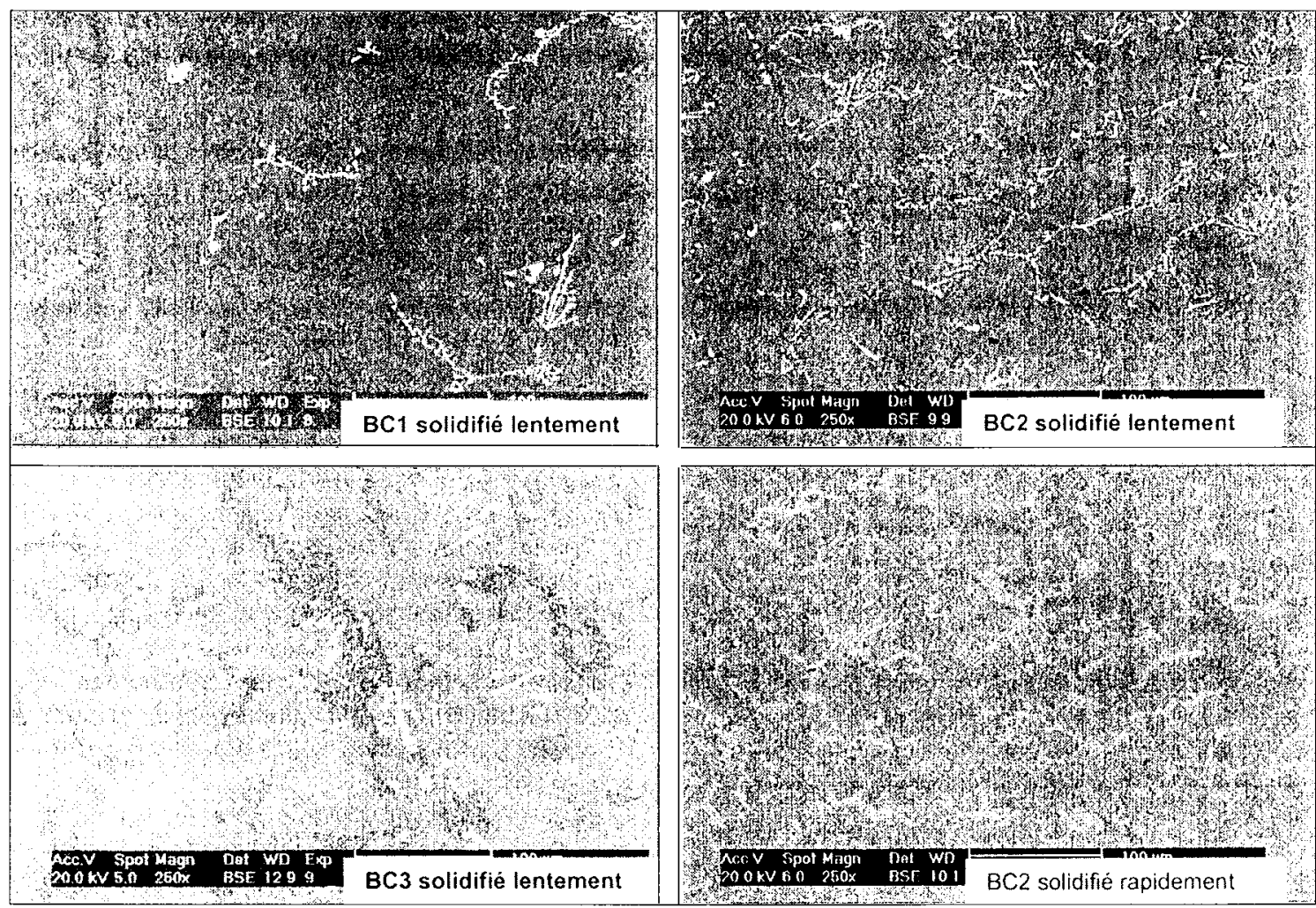

Figure 2 - Exemples de microstructures obtenues (BC1 et $\mathrm{BC} 3$ solidifiés en moule de sable, $B C 2$ solidifié en moule de sable et en creuset froid).

Figure 2 - Some examples of the obtained microstructures (BC1 and BC3 solidified in a sand mould, $B C 2$ solidified in a cold crucible).

près de l'analyse EDS). La fraction surfacique apparente des carbures fait penser qu'il en a été de même pour l'élément carbone. Le contrôle de l'homogénéité de composition chimique par analyse EDS n'a pas non plus révélé de différence sensible sur des coupes de pièces solidifiées en moule de sable ou en creuset froid. Seule la finesse microstructurale du premier millimètre d'épaisseur à partir de l'extérieur de la pièce était différente de celle du reste de l'alliage.

\section{Essais thermogravimétriques}

Dix essais thermogravimétriques ont été réalisés à $1000^{\circ} \mathrm{C}$ sur chaque alliage pour les deux microstructures fine et grossière, et à $1100^{\circ} \mathrm{C}$ spécifiquement dans le cas de l'alliage $\mathrm{BC} 2$, toujours pour les deux tailles de grains. 1000 et $1100^{\circ} \mathrm{C}$ représentent le domaine de températures dans lequel de tels alliages sont utilisés en pratique et où ils sont affectés par l'oxydation de façon sensible. La figure 3 montre quelques-unes des courbes obtenues. Les courbes ne sont pas toutes entièrement paraboliques, suite à l'existence de régimes d'oxydation transitoires. Ces derniers se caractérisent par une oxydation initiale assez rapide et souvent linéaire, préalable au régime permanent qui est, lui, parabolique. II arrive aussi que, sous l'effet des contraintes internes de croissance, la couche d'oxyde extérieure se décolle loca- lement de la surface de l'alliage, cette dernière se trouvant à ce moment-là de nouveau exposée à l'oxydation directe. Dans ce cas, on observe une variation importante de gain de masse puis un retour vers un régime d'allure parabolique (la surface n'est plus couverte par une couche d'oxyde d'épaisseur homogène). Les valeurs des constantes paraboliques $\mathrm{Kp}$ obtenues et des rapports de constantes $<$ microstructure fine / microstructure grossière > sont présentées dans le tableau 1 .

TABLEAU II - Valeurs de la constante parabolique d'oxydation obtenues pour tous les essais.

TABLE II - Values of the oxidation parabolic constants obtained for all trials.

\begin{tabular}{|c|c|c|c|}
\hline $\begin{array}{c}\mathrm{Kp} \\
\left(10^{-12} \cdot \mathrm{g}^{2} \cdot \mathrm{cm}^{-4} \cdot \mathrm{s}^{-1}\right)\end{array}$ & $\begin{array}{c}\text { Structure } \\
\text { grossière } \mathrm{Kp}\end{array}$ & $\begin{array}{c}\text { Structure } \\
\text { fine } \mathrm{Kp}_{\mathrm{fin}}\end{array}$ & $\begin{array}{c}\text { Rapport } \\
\mathrm{Kp}_{\mathrm{fin}} / \mathrm{Kp}_{\mathrm{gros}}\end{array}$ \\
\hline $\mathrm{BN} 1\left(1000^{\circ} \mathrm{C}\right)$ & 0,58 & 1,55 & 2,67 \\
$\mathrm{BC} 1\left(1000^{\circ} \mathrm{C}\right)$ & 1,15 & 1,58 & 1,37 \\
$\mathrm{BC} 2\left(1000^{\circ} \mathrm{C}\right)$ & 2,69 & 1,42 & 0,53 \\
$\mathrm{BC} 2\left(1100^{\circ} \mathrm{C}\right)$ & 19,8 & 16,4 & 0,83 \\
$\mathrm{BC} 3\left(1000^{\circ} \mathrm{C}\right)$ & 3,46 & 1,39 & 0,40 \\
\hline
\end{tabular}



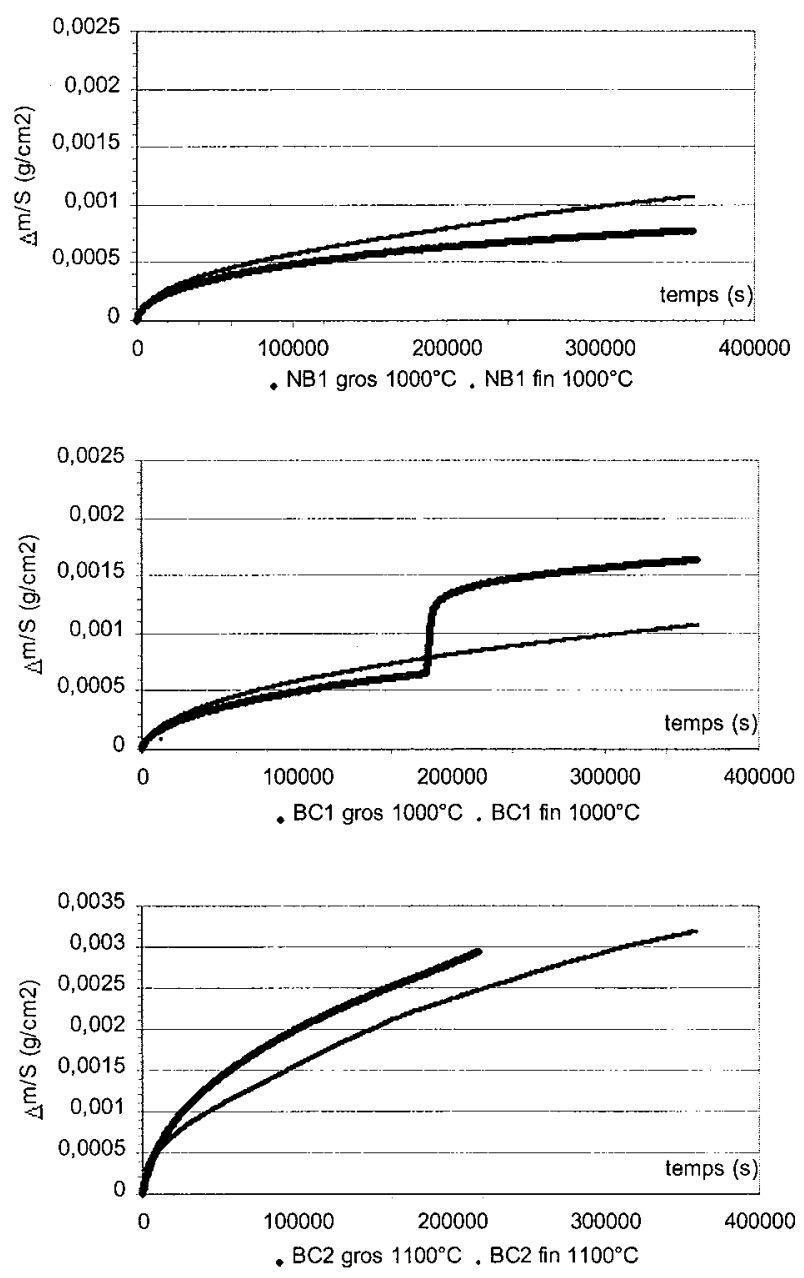

Figure 3 - Courbes thermogravimétriques obtenues sur trois des quatre alliages pour les microstructures grossières et fines.

Figure 3 - Mass gain curves obtained on three of the four alloys for the coarse and fine microstructures.

II apparaît que, sur les courbes comme au niveau des constantes de vitesse, la cinétique d'oxydation à $1000^{\circ} \mathrm{C}$ des alliages est, à composition chimique constante, différente selon la taille de grains. Comparées aux microstructures grossières, les microstructures fines conduisent à une oxydation soit plus lente soit plus rapide, suivant l'alliage considéré. La microstructure fine conduit à l'oxydation la plus rapide dans le cas des alliages BN1 et BC1. Pour les alliages $\mathrm{BC} 2$ et $\mathrm{BC} 3$, c'est au contraire la microstructure grossière qui conduit à l'oxydation la plus rapide. Par contre, cette différence s'amenuise pour l'alliage BC2 lorsque la température est de $1100^{\circ} \mathrm{C}$. On peut remarquer que les alliages dont la vitesse d'oxydation est plus rapide lorsque la microstructure est fine, présentent la particularité de contenir du tungstène dans leur composition chimique, alors que cet élément n'est pas présent dans les autres alliages.

Si on compare les alliages entre eux, lorsque la microstructure est grossière, c'est l'alliage à base de nickel qui s'oxyde le moins rapidement. En ne considérant que les super- alliages à base de cobalt, il apparaît une augmentation de la constante parabolique d'oxydation en fonction de la teneur en carbone de l'alliage. Par contre, dès que les alliages ont une microstructure fine, leurs comportements en oxydation sont très proches les uns des autres. Leurs courbes thermogravimétriques sont quasiment superposables et les constantes $\mathrm{Kp}$ presque identiques (pour tous les alliages les constantes Kp restent entre 1,4 et $1,6 \cdot 10^{-12} \mathrm{~g}^{2} \cdot \mathrm{cm}^{-4} \cdot \mathrm{s}^{-1}$ ).

\section{Examen métallographique des surfaces oxydées}

Après le test thermogravimétrique, les plaquettes ont été observées au niveau de la zone affectée par l'oxydation (fig. 4). D'une manière générale, il a été possible d'examiner la couche externe d'oxydes au moins localement et d'estimer son épaisseur (sauf dans les cas où la desquamation au refroidissement après essai a été totale). La partie de l'alliage en extrême surface touchée par l'oxydation interne (les premiers $100 \mu \mathrm{m}$ ) ou par des modifications microstructurales consécutives à l'évolution locale de la composition chimique due à l'oxydation, a également été observée.

L'alliage BN1 a partiellement perdu sa couche d'oxyde externe, que sa microstructure soit fine ou grossière. Dans les deux cas, son épaisseur vaut à peu près $5 \mu \mathrm{m}$. La couche est constituée majoritairement de chromine $\mathrm{Cr}_{2} \mathrm{O}_{3}$ mais contient aussi par endroit des petits oxydes plus clairs dont l'analyse a été difficile mais a révélé la présence de tungstène (environ $8 \%$ massique ou $2 \%$ atomique). Par endroit, les carbures intergranulaires ont disparu sur environ $20 \mu \mathrm{m}$ en laissant des cavités ayant conservé leurs formes. On ne note pas de réelle différence au niveau des phénomènes microstructuraux entre les deux finesses de microstructure.

L'alliage BC1 avec microstructure grossière a lui aussi conservé par endroits la couche d'oxyde externe, d'une épaisseur de $5 \mu \mathrm{m}$ environ. Là aussi l'examen au MEB en mode BSE et l'analyse EDS ponctuelle montrent que la couche de chromine contient localement d'autres petits oxydes. Par contre, en microstructure fine, la couche d'oxyde externe est entièrement partie par desquamation lors du retour à la température ambiante. L'oxydation a été également interne sur une profondeur de $20 \mu \mathrm{m}$ depuis l'extrême surface. Les carbures $(\mathrm{Cr}, \mathrm{W})_{23} \mathrm{C}_{6}$ sont remplacés par des oxydes $\mathrm{Cr}_{2} \mathrm{O}_{3}$ et des vides. Les carbures TaC sont remplacés intégralement par des oxydes de chrome et tantale $\mathrm{CrTaO}_{4}$ (identifiés au MEB en analyse EDS ponctuelle) occupant tout le volume laissé par les carbures.

La couche externe d'oxyde de l'alliage BC3 est assez bien conservée. Elle n'est apparemment faite que de chromine et a une épaisseur d'environ $10 \mu \mathrm{m}$ quand sa microstructure est grossière. Quand la microstructure de l'alliage est fine, la couche de chromine est très fragmentée et ne se prête pas à une mesure d'épaisseur (elle paraît cependant avoir été assez épaisse). II y a eu aussi oxydation interne, sur environ $15 \mu \mathrm{m}$ pour la structure grossière et plutôt $10 \mu \mathrm{m}$ pour la structure fine : dans les deux cas, oxydation des carbures de 

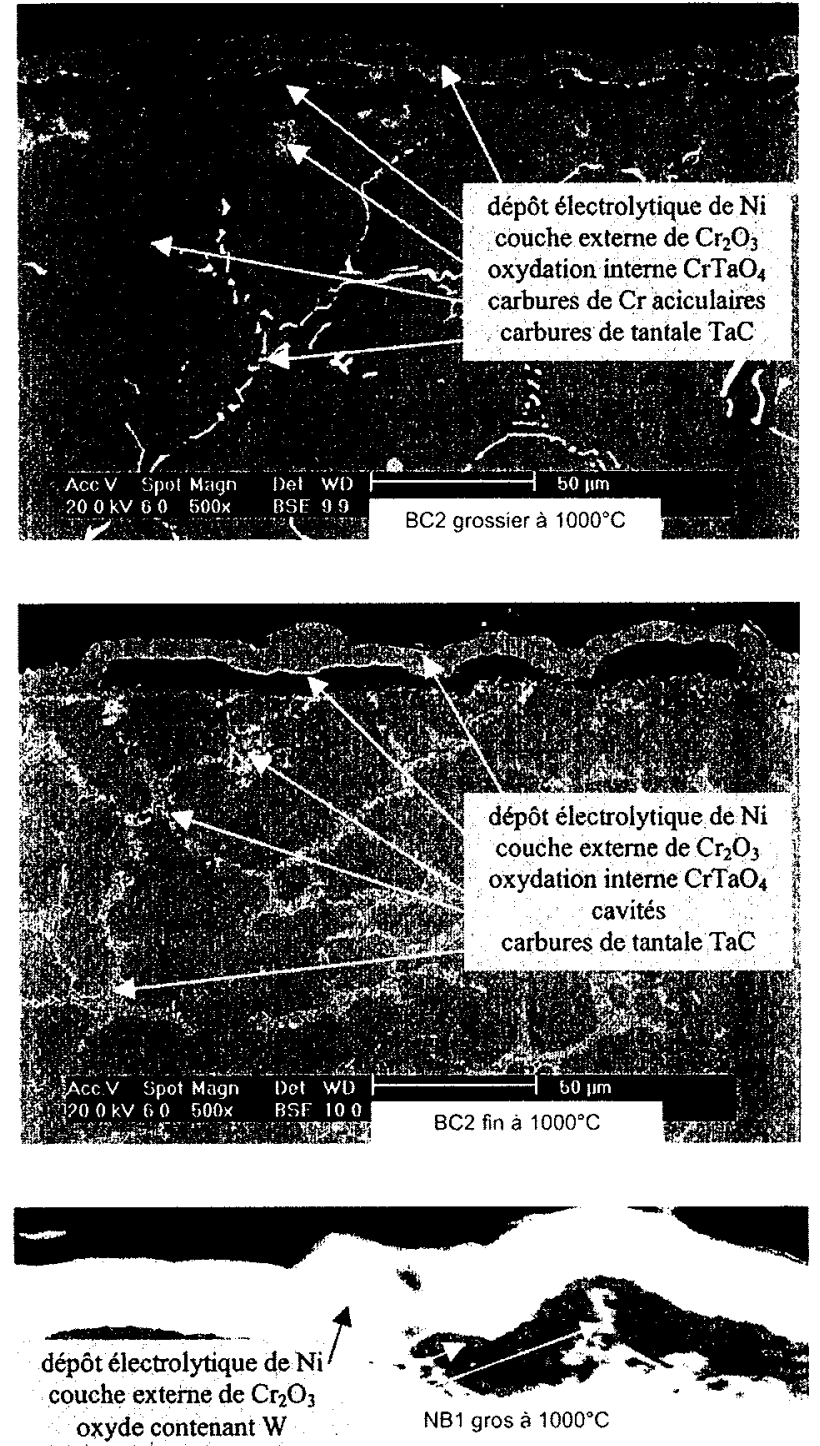

Figure 4 - État de surface après oxydation durant $100 \mathrm{~h}$ à $1000^{\circ} \mathrm{C}$ (alliage $\mathrm{BC} 2$ en structure grossière et en structure fine ; détails de la couche d'oxyde externe de NB1 en structure grossière).

Figure 4 - Surface state after oxidation at $1,000^{\circ} \mathrm{C}$ for $100 \mathrm{~h}$ (here : $B C 2$ with coarse and fine microstructures; details of the external oxide scale of NB1).

tantale majoritairement, mais aussi des carbures de chrome dans le cas de la structure fine. Pour le BC3 à structure fine, on note de plus une apparition généralisée de cavités sur $15 \mu \mathrm{m}$ environ. L'alliage BC3 grossier est, lui, spécifiquement sujet à l'apparition de précipités aciculaires orientés dans une zone de plusieurs dizaines de micromètres vers l'intérieur, séparée de l'extrême surface par une zone décarburée de $15 \mu \mathrm{m}$ environ.

L'alliage $\mathrm{BC} 2$ oxydé à $1000^{\circ} \mathrm{C}$ présente une couche de chromine assez parcellaire. Elle semble avoir été d'environ $6 \mu \mathrm{m}$ d'épaisseur pour la structure grossière comme pour la structure fine. Dans les deux cas, l'oxydation interne ne concerne que les carbures $\mathrm{TaC}$ et affecte une profondeur de $20 \mu \mathrm{m}$, avec une légère tendance à la formation de cavités pour la structure fine. Comme l'alliage $\mathrm{BC} 3$, l'alliage $\mathrm{BC} 2$ - avec structure grossière uniquement - est le siège d'une précipitation de carbures de chrome aciculaires dans la matrice. Après oxydation à $1100^{\circ} \mathrm{C}$, l'alliage $\mathrm{BC} 2$ présente une couche de chromine d'environ $10 \mu \mathrm{m}$ pour la structure grossière ainsi que pour la structure fine. La profondeur affectée par l'oxydation est plus grande qu'à $1000^{\circ} \mathrm{C}$, avec oxydation interne du tantale et également du chrome, et présence de cavités. Cette zone apparaît plus exempte de carbures initiaux qu'après oxydation à $1000^{\circ} \mathrm{C}$. Globalement, bien que difficiles à estimer précisément, les épaisseurs de chromine externe observées semblent à peu près en accord avec les constantes Kp correspondantes.

\section{Discussion}

La taille microstructurale intervient de façon assez sensible sur la cinétique d'oxydation de ces superalliages à base de nickel ou de cobalt, ainsi que sur les phénomènes associés se produisant au niveau de la microstructure sub-corticale. Pour tous les alliages testés à $1000^{\circ} \mathrm{C}$, une différence a pu être relevée entre microstructure grossière et microstructure fine pour une même composition chimique. Par exemple, BC1 s'oxyde plus rapidement à $1000^{\circ} \mathrm{C}$ lorsque sa microstructure est fine que lorsqu'elle est grossière. Cela avait déjà été constaté sur un alliage similaire (8), mais à $1150^{\circ} \mathrm{C}$ où la structure fine conduisait à un doublement de la constante parabolique : $\mathrm{Kp}=59.10^{-12} \mathrm{~g}^{2} \mathrm{~cm}^{-4} \cdot \mathrm{s}^{-1}$ contre $31.10^{-12} \mathrm{~g}^{2} \cdot \mathrm{cm}^{-4} \cdot \mathrm{s}^{-1}$ pour la structure grossière (valeurs corrigées de la volatilisation de la chromine : $\left.K v=12,5 \cdot 10^{-10} \mathrm{~g} \cdot \mathrm{cm}^{-2} \cdot \mathrm{s}^{-1}\right)$. Suivant les alliages, l'écart est dans un sens ou dans l'autre et il semble que le tungstène joue un rôle important. II semble en effet conditionner le sens d'évolution de la vitesse d'oxydation lorsqu'on affine la microstructure. Pour tenter d'expliquer ce qui a pu être observé, on peut rappeler le rôle particulier des joints de grains sur le plan diffusionnel pour différentes espèces impliquées dans le phénomène d'oxydation (le chrome par exemple). De plus nombreux joints de grains émergeant par unité de surface peuvent permettre un approvisionnement de la surface en chrome, et donc une constitution plus rapide et un entretien plus efficace de la couche de chromine. Dans cette hypothèse, cette dernière peut être moins perméable à la diffusion des ions $\mathrm{O}^{2-}$ ou des cations métalliques, d'où un abaissement de la cinétique lorsque la microstructure est plus fine (alliages $\mathrm{BC} 2$ et BC3). Par contre, lorsque l'alliage contient aussi du tungstène (alliages BN1 et BC1), qui est ici partiellement présent aux joints de grains au sein des carbures $M_{23} C_{6}$, ce dernier peut diffuser vers l'extérieur et perturber la couche de chromine en participant localement à la formation d'un oxyde mixte. Cette couche étant alors moins protectrice, il peut s'ensuivre une vitesse d'oxydation augmentée.

Pour les superalliages à base de cobalt, une croissance de la constante $\mathrm{Kp}$ en fonction de la teneur en carbone de l'alliage, donc de la densité des carbures intergranulaires quelles que soient leurs natures, a pu être observée. C'est un fait qui avait déjà été constaté antérieurement (8) sur des superalliages de fonderie similaires. Par contre, les ciné- 
tiques d'oxydation de tous les alliages deviennent quasiment égales lorsque leurs microstructures sont fines. Cela amène à penser qu'une taille microstructurale de $20 \mu \mathrm{m}$ rend la vitesse d'oxydation moins dépendante des quantités et natures de carbures. Dans le cas de $\mathrm{BC} 2$, l'élévation de la température d'essai de 1000 à $1100^{\circ} \mathrm{C}$ semble avoir un peu le même effet, en rapprochant les cinétiques d'oxydation des microstructures grossière et fine. D'un point de vue métallographique, on a pu constater que les produits d'oxydation pouvaient être diversifiés, avec l'oxydation sélective externe (couche de chromine) et interne du chrome et du tantale. Les carbures de chrome et de tantale les plus proches de la surface ont ainsi souffert de l'oxydation. Suivant les cas, les carbures de chrome sont oxydés sur place ou disparaissent totalement, en laissant des cavités dans les deux cas. Ce comportement des carbures de chrome dépend de l'alliage et parfois de la finesse microstructurale, dépendance qui n'apparaît pas facile à expliquer. Les carbures de tantale semblent surtout s'oxyder sur place en formant un oxyde ne laissant aucune cavité. Par contre, l'alliage $\mathrm{BC} 2$ à $1100^{\circ} \mathrm{C}$ est caractérisé par l'apparition d'une fine zone décarburée qui a perdu les carbures de tantale qu'elle contenait, sans laisser de cavités. Des travaux antérieurs (7) ont montré pour un alliage similaire, qu'en élevant encore la température d'essai (expositions de $20 \mathrm{~h}$ à $1300^{\circ} \mathrm{C}$ à l'air), cette zone dépourvue de carbures comme de cavités (et d'oxydes internes sauf en extrême surface) devient plus profonde. Elle est alors aussi d'autant plus étendue que la microstructure est fine $(125 \mu \mathrm{m}$ pour la structure grossière et $275 \mu \mathrm{m}$ pour la structure fine). À cette température plus élevée, la finesse microstructurale apparaît alors d'importance sur la vitesse de développement de cette zone décarburée : une microstructure fine semble alors favoriser la diffusion du tantale vers le front d'oxydation plutôt que l'oxydation interne.

Enfin, il a pu être signalé l'apparition, dans la matrice proche de la surface, de carbures de chrome aciculaires pour les alliages $\mathrm{BC} 2$ et $\mathrm{BC} 3$ à $1000^{\circ} \mathrm{C}$, et ce uniquement dans le cas des structures grossières. Un tel phénomène avait déjà été remarqué et expliqué antérieurement (9) sur des alliages proches après oxydation à $1000^{\circ} \mathrm{C}$. Une interprétation en avait alors été proposée : le carbone libéré par les carbures au cours de la décomposition provoquée par la diffusion de l'élément métallique constitutif ( $\mathrm{Cr}$ ou $\mathrm{Ta}$ ) peut dans certaines conditions réintégrer la matrice, et y provoquer la précipitation de carbures avec le chrome présent en solution solide. Au sujet de ce phénomène-là aussi, la taille microstructurale semble avoir un rôle.

\section{ÉTUDE DE L'INFLUENCE DE L'ORIENTATION DENDRITIQUE}

\section{Détails expérimentaux}

Dans cette deuxième partie, seuls les alliages BN1 et $\mathrm{BC} 1$ ont été considérés. Ces alliages ont été élaborés de la même manière que dans la partie précédente (pièces de fonderie de taille caractéristique $30 \mathrm{~mm}$ ). Par contre, le mode de prélèvement et de préparation des plaquettes pour les essais thermogravimétriques est spécifique. Les pièces
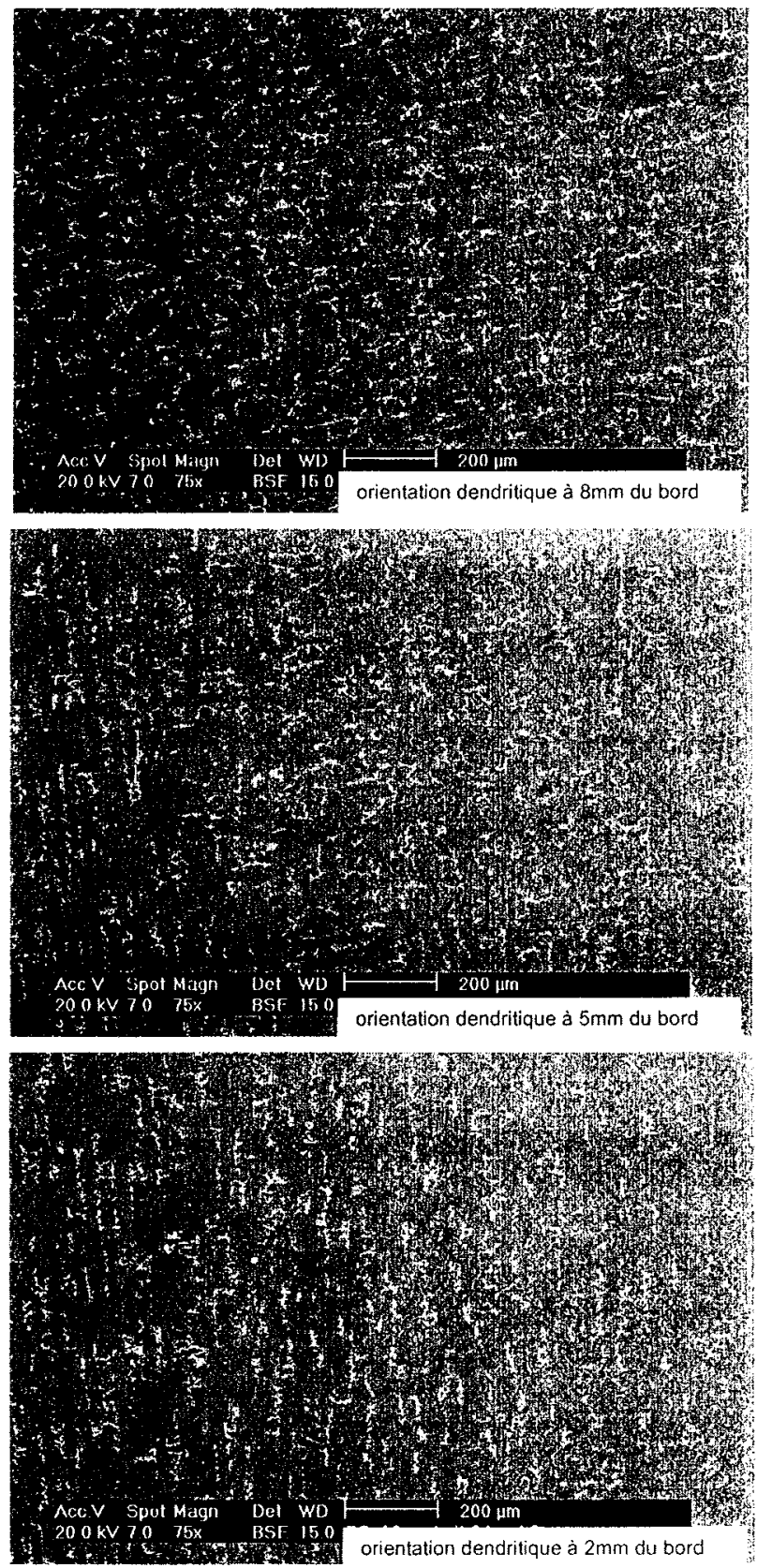

Figure 5 - Evolution de l'orientation dendritique avec la distance depuis le bord de la pièce.

Figure 5 - Evolution of the dendritic orientation with a growing distance from the cast piece surface.

brutes présentent une orientation dendritique, globalement perpendiculaire à la surface extérieure locale de la pièce de fonderie. Cette orientation existe plus loin dans l'alliage jusqu'à, grosso modo, un centimètre de la surface. Plus loin, cette orientation préférentielle fait place à une structure plutôt équiaxe. Cette évolution est illustrée par la figure 5 . Les plaquettes, de même forme et de mêmes dimensions que dans la partie précédente, ont été prélevées de façon à privilégier l'une ou l'autre des deux orientations sachant que les six faces rectangulaires n'ont pas la même orientation 
par rapport aux dendrites. L'homogénéité de microstructure et de taille a été contrôlée dans la plaquette entière. Les plaquettes ont été découpées suivant le plan de prélèvement indiqué dans la figure 6 . Chaque plaquette présente donc une portion de surface globalement parallèle aux dendrites $\left(S_{/ /}\right)$et une surface perpendiculaire aux dendrites $\left(S_{\perp}\right)$, l'une étant prépondérante sur l'autre et leur somme étant égale à la surface extérieure totale $\left(S=S_{\| \prime}\right.$ $+S_{\perp}$ ). $S_{/ /}$et $S_{\perp}$ sont chacune la somme des surfaces élémentaires des différentes faces concernées par l'orientation considérée. Dans le cas où l'un des petits côtés se retrouve dans une zone d'orientation plus aléatoire (proche de la zone équiaxe), sa surface est considérée comme étant composée pour moitié de surface parallèle aux dendrites et pour moitié de surface qui leur est perpendiculaire.

La cinétique d'oxydation de la plaquette, qui suit la loi parabolique [2], est en fait la superposition des lois :

$$
\frac{\Delta \mathrm{m}_{/ /}}{\mathrm{S}_{/ /}}(\mathrm{t})=\sqrt{2 \cdot \mathrm{Kp} / /} \cdot \mathrm{t}^{\frac{1}{2}}
$$

et

$$
\frac{\Delta \mathrm{m}_{\perp}}{\mathrm{S}_{\perp}}(\mathrm{t})=\sqrt{2 . \mathrm{Kp}_{\perp}} \cdot \mathrm{t}^{\frac{1}{2}}
$$

Lorsque la phase d'oxydation de cinétique parabolique est suffisante et lorsque les contributions des deux types de surfaces suivent bien des lois paraboliques, la cinétique d'oxydation est représentée par l'équation [5], qui conduit à la forme réduite [6]

$$
\begin{gathered}
\frac{\Delta \mathrm{m}}{\mathrm{S}}(\mathrm{t})=\frac{\Delta \mathrm{m}_{/ /}(\mathrm{t})+\Delta \mathrm{m}_{\perp}(\mathrm{t})}{\mathrm{S}_{/ /}+\mathrm{S}_{\perp}}=\frac{\mathrm{S}_{/ /}}{\mathrm{S}_{/ /}+\mathrm{S}_{\perp}} \sqrt{2 \cdot \mathrm{Kp}_{/ /}} \cdot \sqrt{\mathrm{t}}+\frac{\mathrm{S}_{\perp}}{\mathrm{S}_{/ /}+\mathrm{S}_{\perp}} \sqrt{2 \cdot \mathrm{Kp} \perp} \cdot \sqrt{\mathrm{t}} \\
\frac{\mathrm{S}_{/ /}}{\mathrm{S}} \sqrt{2 \cdot \mathrm{Kp} / /}+\frac{\mathrm{S}_{\perp}}{\mathrm{S}} \sqrt{2 \cdot \mathrm{Kp}_{\perp}}=\sqrt{2 \cdot \mathrm{Kp}}
\end{gathered}
$$

L'équation [6] est une équation linéaire à deux inconnues $X=\sqrt{2 . K p} / /$ et $Y=\sqrt{2 . K p_{\perp}}$. Pour un même alliage et une même température d'essai, elle s'applique aux deux plaquettes d'orientations différentes (avec les deux valeurs de $\mathrm{Kp}$ obtenues), ce qui conduit à un système linaire de deux équations à deux inconnues $X$ et $Y$, dont la résolution permet d'extraire les valeurs de $\mathrm{Kp}_{/ /}$et $\mathrm{Kp} \mathrm{p}_{\perp}$.

Pour les essais à 1050 et $1100^{\circ} \mathrm{C}$, la détermination des constantes $\mathrm{Kp}$ a été réalisée sur les fichiers de mesures une fois ceux-ci corrigés de la volatilisation de la chromine suivant la même procédure que dans la première partie.

\section{Essais thermogravimétriques}

Huit essais thermogravimétriques ont été réalisés. Les alliages $\mathrm{BN} 1$ et $\mathrm{BC} 1$ ont été testés à $1000^{\circ} \mathrm{C}$ pour les deux orientations. Il en a été fait de même à plus haute température : $\mathrm{BN} 1$ à $1100^{\circ} \mathrm{C}$ et $\mathrm{BC} 1$ à $1050^{\circ} \mathrm{C}$. La figure 7 montre à titre d'exemple les courbes obtenues pour $1000^{\circ} \mathrm{C}$ et le tableau I/I présente l'ensemble des constantes Kp mesurées sur les courbes $\left(\mathrm{Kp}_{\text {plaq// }}\right.$ et $\left.\mathrm{Kp}_{\text {plaq } \perp}\right)$ ainsi que les valeurs découplées des constantes associées aux deux types d'orientation.

Pour l'alliage BN1, il existe une différence entre les deux orientations, avec une oxydation sensiblement plus rapide pour la plaquette majoritairement parallèle aux dendrites que pour celle qui leur est majoritairement perpendiculaire. Cette
TABLEAU III - Valeurs des constantes paraboliques d'oxydation mesurées et valeurs spécifiques pour les deux orientations obtenues par découplage des deux contributions.

TABLE III - Values of the oxidation parabolic constants

\begin{tabular}{|c|c|c|c|c|c|c|}
\hline $1000^{\circ} \mathrm{C}$ & $\mathrm{Kp}_{\text {plaq } / /}$ & $\mathrm{Kp}_{/ /}$ & \multirow{6}{*}{$\begin{array}{c}\text { Constantes } \\
\mathrm{Kp} \\
\left(10^{-12} \mathrm{~g}^{2} \cdot \mathrm{cm}^{-4} \cdot \mathrm{s}^{-1}\right)\end{array}$} & $>1000^{\circ} \mathrm{C}$ & $\mathrm{Kp}_{\text {plaq } / /}$ & $\mathrm{Kp}_{/}$ \\
\hline Alliages & $\mathrm{KP} P_{\text {plaq } \perp}$ & $K p_{\perp}$ & & Alliages & $\mathrm{KP}_{\text {plaq } \perp}$ & $\mathrm{Kp} \mathrm{p}_{\perp}$ \\
\hline \multirow{2}{*}{ BN1 } & 1,71 & 2,30 & & BN1 & 7,12 & 8,20 \\
\hline & 0,63 & 0,19 & & $\left(1100^{\circ} \mathrm{C}\right)$ & 3,86 & 2,40 \\
\hline \multirow{2}{*}{$\mathrm{BC} 1$} & 1,32 & 1,22 & & $\mathrm{BC} 1$ & 3,60 & 3,32 \\
\hline & 1,69 & 1,90 & & $\left(1050^{\circ} \mathrm{C}\right)$ & 5,11 & 5,71 \\
\hline
\end{tabular}
obtained on the curves and of the specific values corresponding to the uncoupled contributions of the two orientations. 


\section{oxydation}
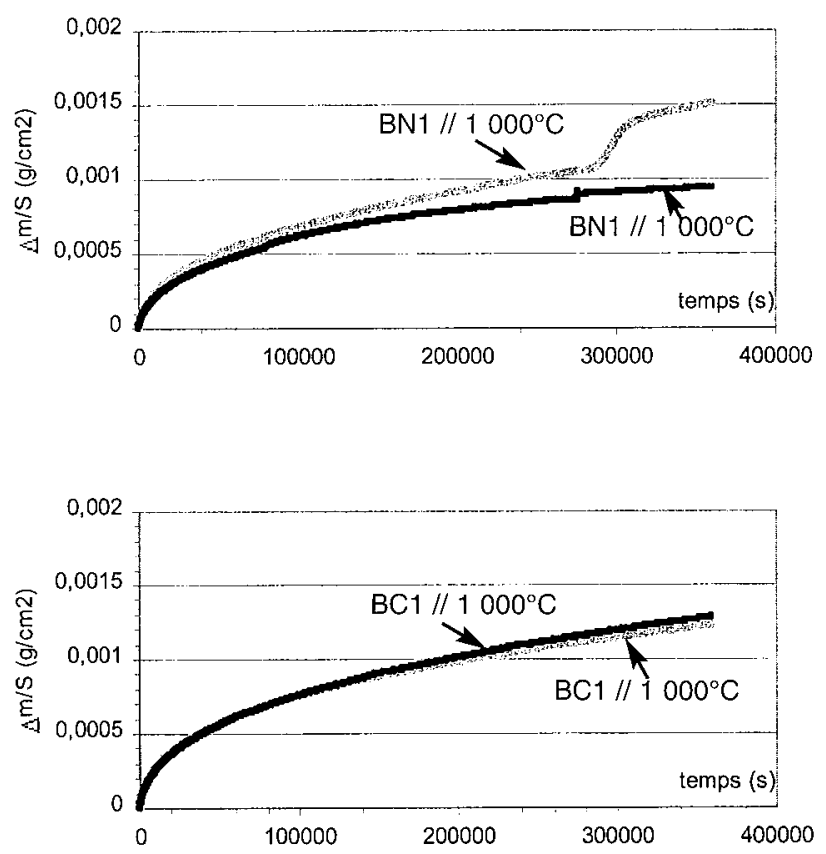

Figure 7 - Courbes thermogravimétriques à $1000^{\circ} \mathrm{C}$ des alliages $\mathrm{BN} 1$ et $\mathrm{BC} 1$ pour les deux orientations.

Figure 7 - Mass gain curves at $1,000^{\circ} \mathrm{C}$ of the $\mathrm{BN} 1$ and $\mathrm{BC} 1$ alloys for the two orientations.

tendance s'affirme pour cet alliage lors des tests à $1100^{\circ} \mathrm{C}$. On remarque aussi que les courbes correspondant à une surface principalement parallèle aux dendrites sont les plus affectées par le décollement de l'oxyde suite à des contraintes de croissance plus sévères, sans que cela ne gêne la détermination des constantes. La cinétique d'oxydation pour le cas parallèle paraît donc être systématiquement supérieure à celle du cas perpendiculaire. Les constantes $\mathrm{Kp}$ globales relevées sur les courbes confirment cette observation. Le découplage des contributions accentue les différences, les valeurs de $\mathrm{Kp}_{/ /}$et $\mathrm{Kp}_{\perp}$ montrant une très grande différence (rapports $\times 12$ à $1000^{\circ} \mathrm{C}$ et $\times 3,4$ à $1100^{\circ} \mathrm{C}$ en faveur de $\left.\mathrm{Kp}_{/ /}\right)$.

Pour l'alliage BC1, la hiérarchie est inversée entre les plaquettes parallèle et perpendiculaire aux dendrites. L'oxydation est plus lente pour la première que pour la seconde (rapports $\times 1,6$ à $1000^{\circ} \mathrm{C}$ et $\times 1,7$ à $1100^{\circ} \mathrm{C}$ en faveur de $\mathrm{Kp} \mathrm{p}_{\perp}$ ).

\section{Examen métallographique des surfaces oxydées}

Après coupe dans le sens adéquat, enrobage et polissage, les plaquettes oxydées ont été observées des points de vue de l'épaisseur d'oxyde externe, de la profondeur de l'oxydation interne et de l'épaisseur de la zone décarburée. Ce sont là des grandeurs locales qui peuvent être directement associées à une orientation particulière sans découplage préalable, à la différence de la prise de masse totale qui au contraire globalise toutes les contributions. Des mesures ont été tentées pour donner un ordre de grandeur à ces caractéristiques de l'oxydation observables par métallographie, avec une difficulté particulière pour la couche de chromine externe dont il ne reste parfois, suite au refroidissement après essai, que quelques fragments.

L'alliage BN1 a globalement assez bien conservé sa couche d'oxyde externe, bien qu'il y ait eu desquamation au refroidissement (fig. 8). Avec la mesure de l'épaisseur de cette couche on retrouve la plus grande rapidité de l'oxydation à $1100^{\circ} \mathrm{C}$ (14 et $8 \mu \mathrm{m}$ sur respectivement les grandes faces $/$ et ${ }_{\perp}$ aux dendrites) qu'à $1000^{\circ} \mathrm{C}$ ( 8 et $6 \mu \mathrm{m}$ ), ainsi que la hiérarchie en termes de constante parabolique d'oxydation observée en thermogravimétrie entre les deux orientations. L'oxydation interne $\left(\mathrm{Cr}_{2} \mathrm{O}_{3}\right)$ de type interdendritique est plus profonde à $1100^{\circ} \mathrm{C}$ (vers $\left.35 \mu \mathrm{m}\right)$ qu'à $1000^{\circ} \mathrm{C}(<20 \mu \mathrm{m})$, mais il est difficile de voir une différence sensible entre les deux orientations. Les deux observations précédentes sont aussi valables pour la profondeur décarburée qui vaut à peu près $35 \mu \mathrm{m}$ à $1100^{\circ} \mathrm{C}$ et environ 10 à $20 \mu \mathrm{m}$ à $1000^{\circ} \mathrm{C}$ quelle que soit l'orientation.
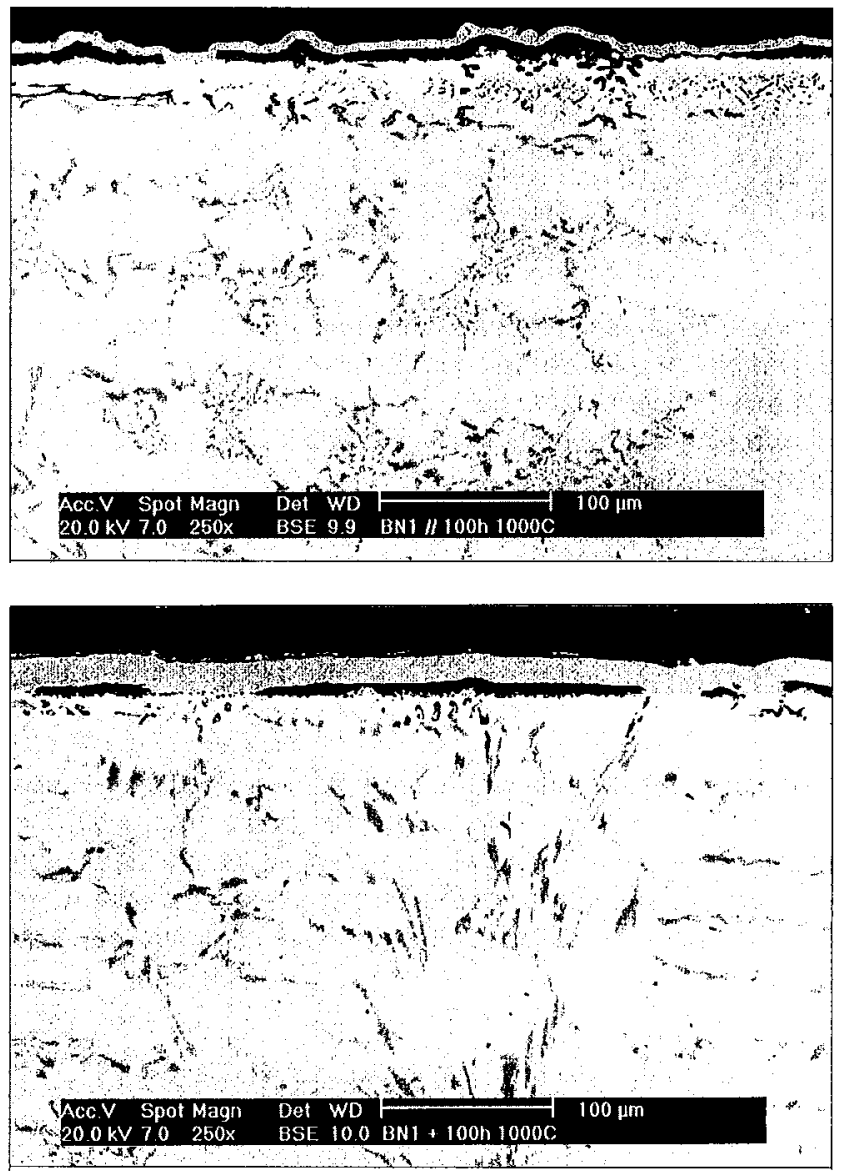

Figure 8 - Front d'oxydation pour l'alliage NB1 après $100 \mathrm{~h}$ à $1000^{\circ} \mathrm{C}$ pour les deux orientations.

Figure 8 - Oxidation front of NB1 alloy after exposure at $1,000^{\circ} \mathrm{C}$ for $100 \mathrm{~h}$ for the two orientations. 

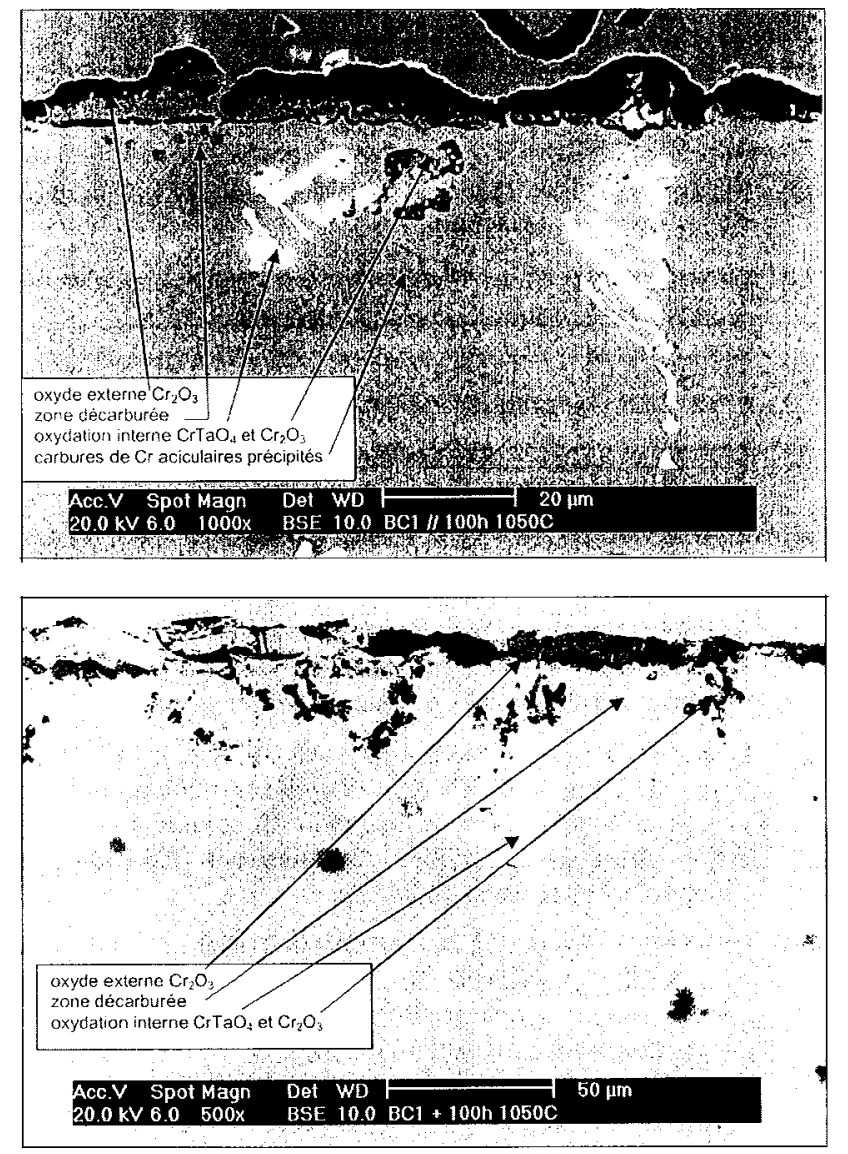

Figure 9 - Front d'oxydation pour l'alliage BC1 après $100 \mathrm{~h}$ à $1050^{\circ} \mathrm{C}$ pour les deux orientations.

Figure 9 - Oxidation front of $B C 1$ alloy after exposure at $1,050^{\circ} \mathrm{C}$ for $100 \mathrm{~h}$ for the two orientations.

L'alliage $\mathrm{BC} 1$ présente une épaisseur de couche d'oxyde externe de $6 \mu \mathrm{m}$ à $1050^{\circ} \mathrm{C}$ et de $4 \mu \mathrm{m}$ à $1000^{\circ} \mathrm{C}$, quelle que soit l'orientation. Ceci est en accord avec le fait que les valeurs de constantes paraboliques des cas parallèle et perpendiculaire sont très proches l'une de l'autre. Profondeur d'oxydation interne et profondeur décarburée ne sont pas sensiblement différentes entre les deux températures. Par contre, elle semblent dépendre de l'orientation : toutes deux tendraient à être plus fortes dans le cas perpendiculaire que dans le cas parallèle aux dendrites (respectivement environ 30 et $20 \mu \mathrm{m}$ ) (fig. 9).

\section{Discussion}

Pour les alliages étudiés ici il existe bien un effet de l'orientation dendritique. L'alliage BN1 est particulièrement sensible à cet effet puisque la constante parabolique dans le cas parallèle est, à $1100^{\circ} \mathrm{C}$, presque 4 fois supérieure à celle du cas perpendiculaire. Ceci correspond à un doublement de l'épaisseur finale si la cinétique est parabolique sur toute la période, ce qui est le cas. $\grave{A} 1000^{\circ} \mathrm{C}$, le rapport est plus grand (facteur 10 ) mais correspond moins aux épaisseurs mesurées. Cette différence des vitesses d'oxydation entre les deux types d'orientation avait déjà été constatée et étudiée sur des alliages à base de nickel simplifiés Ni-Cr-C possédant deux niveaux de densité de carbures de chrome aux joints de grains. $\dot{A} 1000$ et à $1100^{\circ} \mathrm{C}$, la cinétique d'oxydation était supérieure dans le cas parallèle comme dans le cas perpendiculaire, mais dans un moindre rapport.

L'alliage BC1 semble être moins sensible à ce phénomène mais une différence entre les deux orientations a tout de même été constatée. Dans le cas de cet alliage, ce serait plutôt les surfaces perpendiculaires aux dendrites qui s'oxyderaient plus vite que les surfaces parallèles. Les résultats de l'observation métallographique suggèrent que ce phénomène serait accompagné plutôt d'une oxydation interne plus profonde que d'une couche d'oxyde externe plus épaisse.

Plusieurs explications sont possibles pour comprendre cette dépendance de la cinétique d'oxydation vis-à-vis de l'orientation microstructurale. Dans le cas de l'alliage BN1, ce serait donc au niveau de la couche de chromine externe qu'existerait une différence. Une première explication possible repose sur le rôle de conducteur diffusionnel particulier des joints de grains, pour l'apport du chrome en surface et pour la constitution et l'entretien de la couche d'oxyde extérieur. Dans le cas où la surface est perpendiculaire aux joints de grains, la diffusion du chrome se trouverait facilitée puisqu'il ne ferait que longer les dendrites. L'approvisionnement de la surface en chrome serait alors mieux assurée, et la couche de chromine formée de meilleure qualité que dans le cas parallèle où le chrome doit diffuser en contournant les dendrites ou les traverser. D'autres explications sont également possibles. Par exemple, lorsque les joints de grains (et donc les carbures intergranulaires) sont orientés (respectivement alignés) parallèlement à la surface, la couche externe de chromine germe et croitrait d'une façon différente à ce qu'elle ferait si l'orientation était perpendiculaire. De fait, elle pourrait posséder des propriétés cristallines spécifiques davantage favorables à une diffusion cationique depuis l'interface métal-oxyde vers l'interface oxyde-air.

Dans le cas de l'alliage $B C 1$, c'est surtout une différence d'oxydation interne interdendritique qui a pu être relevée. Pour expliquer la profondeur d'oxydation interne du BC1 différente suivant l'orientation, plusieurs hypothèses peuvent être énoncées. Par exemple, les propriétés cristallines de la couche d'oxyde externe, comme évoqué précédemment mais cette fois-ci en faveur de l'orientation perpendiculaire, peuvent aussi être davantage favorables à une diffusion des espèces ioniques $\mathrm{O}^{2-}$ au travers des joints de grains de l'alliage en traversant l'oxyde interne déjà formé. Plus simplement, il est possible aussi que pour les ions $\mathrm{O}^{2-}$ ou pour l'air, le parcours étant plus direct dans le cas perpendiculaire, la profondeur atteinte au bout d'un même temps serait plus profonde pour une même longueur de parcours (dans le cas parallèle le parcours diffusionnel serait plus compliqué). Hélas, la perte systématique au refroidissement d'une bonne partie de la couche d'oxyde externe empêche une caractérisation de cette couche après essai. 
La façon, entre les deux alliages, suivant laquelle la cinétique d'oxydation dépend de l'orientation, peut tenir à plusieurs facteurs : l'élément de base (Ni ou Co pour la diffusion du chrome au travers de la matrice), la densité des carbures interdendritiques (plus élevée dans le cas de BN1 que dans celui de $\mathrm{BC} 1$ ) et la nature de ces carbures (présence de carbures $\mathrm{TaC}$ dans $\mathrm{BC} 1$ en supplément des carbures de chrome).

\section{- CONCLUSIONS}

Les effets de la finesse microstructurale et de l'orientation dendritique des superalliages de fonderie sur leur cinétique d'oxydation à haute température existent manifestement. Ils semblent cependant ne pas avoir été beaucoup étudiés antérieurement, à la différence de leur influence sur les propriétés mécaniques par exemple. Les résultats thermogravimétriques ont précisé les valeurs des constantes paraboliques et leurs écarts entre finesses microstructurales différentes et entre orientations dendritiques différentes. La mise en correspondance de ces données cinétiques avec les étendues de l'oxydation externe et de l'oxydation interne a permis de préciser ce que signifient ces différences de cinétique d'un point de vue métallographique, puis de proposer de premières explications. Le caractère de chemins diffusionnels privilégiés qu'ont les joints de grains fait très probablement que la densité et l'orientation de ces derniers jouent un rôle important sur la diffusion du chrome ou de l'oxygène, et donc sur les caractéristiques de l'oxydation interne et de la couche d'oxydes externe. Hélas, il s'est avéré que cette dernière est fréquemment perdue au refroidissement dans le cas de ces alliages, ce qui n'a pas permis pour le moment des analyses plus poussées.

D'un point de vue pratique, c'est surtout la finesse microstructurale qui représente une possibilité d'améliorer la résistance à l'oxydation d'une pièce de fonderie. En effet, des possibilités multiples existent pour augmenter ou diminuer la vitesse de refroidissement pendant la solidification. Par contre, agir sur l'orientation dendritique, pour des pièces de fonderie classique dont la surface extérieure est le plus souvent perpendiculaire aux dendrites formées, serait plus difficile. Seul un usinage particulier tel que le tronçonnage ou le rainurage, est susceptible de générer localement des surfaces parallèles aux dendrites.

\section{bibliographie}

(1) KOFSTAD (P.) - High temperature corrosion. Elsevier applied science (1988).

(2) KURZ (W.), FISHER (D.J.) - Fundamentals of solidification Trans. Tech. Publ. (1989).

(3) LESOULT (G.) - Solidification. Macrostructures et qualité des produits. M59. Techniques de I'Ingénieur (1989).

(4) LESOULT (G.) - Solidification. Cristallisation et microstructures. M58. Techniques de I'Ingénieur (1986)

(5) BRADLEY (E.F.) - Superalloys : a technical guide. ASM International (1988).

(6) SIMS (C.T.), HAGEL (W.C.) - The superalloys. John Wiley \& Sons (1972).

(7) DI MARTINO (J.) - Oxydation à haute température et corrosion par le verre $\mathrm{C} 3$ de superalliages base cobalt. Thèse de Doctorat de l'Université de Nancy 1 (2002).

(8) DI MARTINO (J.), MICHON (S.), ARANDA (L.), BERTHOD (P.), PODOR (R.), RAPIN (C.) - Oxydation à haute température et corrosion par le verre fondu de superalliages base cobalt. Annales de Chimie - Science des Matériaux, 28 (Suppl. 1) (2003), S231-S238.

(9) BERTHOD (P.), MICHON (S.), DI MARTINO (J.), MATHIEU (S.), NOEL (S.), PODOR (R.), RAPIN (C.) - Thermodynamic calculations for studying high temperature oxidation of superalloys. Calphad, $27 / 3$ (2003), p. 279-288. 